\title{
Enhanced and Reduced Atom Number Fluctuations in a BEC Splitter
}

\author{
Kenneth Maussang, ${ }^{1}$ G. Edward Marti, ${ }^{1 *}$ Tobias Schneider,${ }^{1, \dagger}$ Philipp Treutlein, ${ }^{2}$ Yun Li, ${ }^{1,3}$ Alice Sinatra, ${ }^{1}$ \\ Romain Long, ${ }^{1}$ Jérôme Estève, ${ }^{1}$ and Jakob Reichel ${ }^{1, \hbar}$ \\ ${ }^{1}$ Laboratoire Kastler-Brossel, ENS, Université Pierre et Marie Curie-Paris 6, CNRS, 24 rue Lhomond, 75231 Paris Cedex 05, France \\ ${ }^{2}$ Departement Physik, Universität Basel, Klingelbergstrasse 82, CH-4056 Basel, Switzerland \\ ${ }^{3}$ Precision Spectroscopy, Department of Physics, East China Normal University, Shanghai 200062, China
}

(Received 11 May 2010; published 19 August 2010)

\begin{abstract}
We measure atom number statistics after splitting a gas of ultracold ${ }^{87} \mathrm{Rb}$ atoms in a purely magnetic double-well potential created on an atom chip. Well below the critical temperature for Bose-Einstein condensation $T_{c}$, we observe reduced fluctuations down to $-4.9 \mathrm{~dB}$ below the atom shot noise level. Fluctuations rise to more than $+3.8 \mathrm{~dB}$ close to $T_{c}$, before reaching the shot noise level for higher temperatures. We use two-mode and classical field simulations to model these results. This allows us to confirm that the supershot noise fluctuations directly originate from quantum statistics.
\end{abstract}

DOI: 10.1103/PhysRevLett.105.080403

PACS numbers: 03.75.Lm, 67.10.Ba, 67.85.-d

Since the achievement of Bose-Einstein condensation (BEC) in dilute atomic gases, different experimental techniques have been developed in order to coherently split a BEC into two spatially separate parts [1-4], with atom interferometry as one of the motivations. Even though BECs are usually in the weakly interacting regime, the interactions between the particles dramatically affect the physics of the splitting. In particular, repulsive interactions limit the phase coherence between the two split parts [5], but also reduce atom number difference fluctuations, giving rise to nonclassical squeezed states [6-10].

In this Letter, we use a purely static magnetic potential created on an atom chip to realize a nonlinear spatial "beam" splitter for a BEC. We investigate the physics of the splitting and focus on atom number fluctuations and the role of temperature. At low temperatures, where the interaction energy dominates, we directly observe number squeezed states with relative population fluctuations $-4.9 \mathrm{~dB}$ below shot noise, as first shown in [10] and indirectly observed in [8]. The two separated but weakly linked parts of the BEC constitute a bosonic Josephson junction, usually described by a two-mode model (TMM) [11]. Our results are in agreement with the TMM, which also predicts that the observed squeezing is accompanied by high phase coherence.

The magnetic trap configuration allows barrier heights up to several $\mu \mathrm{K}$ and straightforward evaporative cooling, so that we can separate clouds with increasing temperature all the way to the nondegenerate regime. In the intermediate temperature regime, where both a significant condensate and thermal fraction are present, we observe large superbinomial fluctuations in the number difference between the two parts. This excess of fluctuations is a direct signature of the Bose statistics, in close analogy to the bunching effect in quantum optics [12].

Close to the BEC transition, the condensates show significant depletion and the TMM breaks down. We comple- ment our experiments by a theoretical investigation of this regime using a classical field approach and show that large superbinomial fluctuations are a general feature at thermal equilibrium. Although the experiments are not performed at equilibrium, our observations are still in qualitative agreement with these theoretical results.

Our experiment uses a two-layer atom chip to prepare a ${ }^{87} \mathrm{Rb} \mathrm{BEC}$ in the $\left|F=2, m_{F}=2\right\rangle$ hyperfine state, and then split it in a double-well potential with adjustable barrier height and well spacing. The magnetic potential along the weak axis is well approximated by $V(x)=V_{b}[1-$ $\left.\left(x / x_{0}\right)^{2}\right]^{2}$, where $V_{b}$ is the barrier height and $2 x_{0}$ is the distance between the wells [Figs. 1(a) and 1(b)]. The transverse potential is harmonic with trap frequencies $\omega_{y, z} / 2 \pi \approx 1 \mathrm{kHz}$. Unlike $\mathrm{rf}$ dressed potentials, which tend to create very elongated traps [3,8], our traps have an aspect ratio close to 1 , which strongly suppresses phase fluctuations within each well. We split the trap by increasing $I_{2}$, which simultaneously increases both $V_{b}$ and $x_{0}$ [Figs. 1(b) and 1(c)]. The interesting regime of two weakly coupled condensates occurs for a barrier height on the order of the chemical potential (few $\mathrm{kHz}$ ), corresponding to a current $I_{2} \simeq 2.5 \mathrm{~mA}$ in our experiments. In this region, the tunneling rate is divided by 2 when the barrier $V_{b} / h$ increases by $75 \mathrm{~Hz}$. We estimate fluctuations of the barrier height to be on the order of $20 \mathrm{~Hz}$. A magnetic field component normal to the chip leads to a small energy difference $\Delta E$ between the two potential minima. A magnetic shield with an attenuation factor of $\sim 30$ reduces fluctuations of $\Delta E$ to a few $\mathrm{Hz}$.

The expected atom number fluctuations are on the order of a few tens of atoms, placing stringent requirements on the imaging system. We perform absorption imaging using a back-illuminated CCD camera and 3 ms delay between absorption and reference images. The total quantum efficiency including optical losses is $q=0.84$. Probe pulses have $\tau=50 \mu$ s duration and an intensity close to satura- 

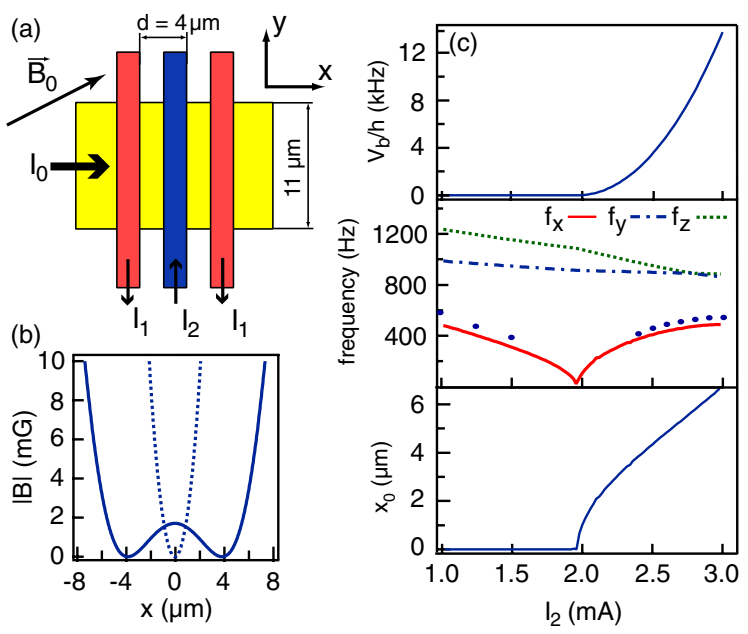

FIG. 1 (color online). (a) Chip geometry. We use dc currents superposed with a static homogeneous field $\mathbf{B}_{\mathbf{0}}=\left(B_{x}, B_{y}, B_{z}\right)=$ $(15,8,0) \mathrm{G}$. To first approximation, the potential can be understood as follows: The field of $I_{0}=100 \mathrm{~mA}$ combines with $\mathbf{B}_{\mathbf{0}}$ to create harmonic confinement in the $x y$ plane. The minimum is located at a distance $z_{0}=\mu_{0} I_{0} / 2 \pi B_{y}$ from the $I_{0}$ wire and the field in the minimum is $B_{x}$. This minimum is modified by $I_{1}=$ $2.0 \mathrm{~mA}$ and $I_{2}$, whose fields are predominantly along $x$ on the trap axis. For $z_{0} \gtrsim d$, the two $I_{1}$ wires together provide harmonic confinement along $x . I_{2}$, of opposite polarity, creates the barrier of adjustable height and also determines the spacing between the two resulting wells. (b) Profile of the trapping potential along the splitting axis $x$, for $I_{2}=0 \mathrm{~mA}$ (cooling trap, dashed line) and $I_{2}=2.4 \mathrm{~mA}$ (solid line). The $x$ axis is the same as in (a). (c) Barrier height $V_{b}$ (top), trap frequencies $\left(f_{x}, f_{y}, f_{z}\right)$ (center), and position $x_{0}$ of the right minimum (bottom) as functions of the current $I_{2}$. Lines, numerical calculation based on current configuration; blue circles, measured frequencies $f_{x}$.

tion. Atom numbers are calibrated following a procedure inspired by [13]. We estimate our total systematic error to be at most $18 \%$. Atoms in the two wells are resolved by applying a large gradient with the central conductor $\left(I_{2}\right)$ for the first $50 \mu \mathrm{s}$ of a $6 \mathrm{~ms}$ time of flight. Photon shot noise, scaling as the root of the image area, leads to a standard deviation of about 20 atoms for a BEC of $10^{3}$ atoms imaged over 800 pixels, and about 60 atoms for a thermal cloud $\left(2 \times 10^{4}\right.$ atoms, 7000 pixels), pixel area being $9.5 \mu \mathrm{m}^{2}$ in the object plane. This is close to the theoretical limit $\sqrt{16 / q \sigma \Gamma \tau}=0.19 \mu \mathrm{m}^{-1}$, where $\sigma$ is the scattering cross section and $\Gamma$ the natural linewidth, and always remains below atom shot noise; furthermore, this noise is well characterized and stable so that it can be subtracted. Technical fluctuations (moving fringes) constitute a second noise source, which is negligible for small BECs, but becomes comparable to photon shot noise for large atomic clouds. We do not attempt to remove this noise, but indicate an estimate of its level (open green dots in Fig. 3).

To measure the fluctuations of the atom number difference $N_{L}-N_{R}$ between the left and the right well, we acquire a data set consisting of a large number of absorp- tion images (typically $>100$ ) taken under identical conditions. In each image, we measure $N_{L}$ and $N_{R}$ (see inset of Fig. 3). To compensate for a slight imbalance in the splitting, we determine the probability to be in the left (right) well $f_{L, R}=\left\langle N_{L, R} / N\right\rangle$, where $N=N_{L}+N_{R}$ and the average is taken over all images. For each image, we calculate $n=f_{R} N_{L}-f_{L} N_{R}$, which is the deviation of $\left(N_{L}-N_{R}\right) / 2$ from the expected value $\left(f_{L}-f_{R}\right)\left(N_{L}+N_{R}\right) / 2$. We define the number squeezing factor as the variance of $z=$ $n / \sqrt{f_{L} f_{R}\left(N_{L}+N_{R}\right)}$. Correcting for the photon shot noise contribution $z_{p}$ [14] leads to the final expression $\xi^{2}=$ $\left\langle z^{2}\right\rangle-\left\langle z_{p}^{2}\right\rangle$. This definition is first-order insensitive to fluctuations in the total number of atoms and produces $\xi^{2}=1$ for a binomial distribution. In our data, the correction $\left\langle z_{p}^{2}\right\rangle$ is always smaller than $\left\langle z^{2}\right\rangle$ itself.

In a first experiment, we split an almost pure BEC of 1300 atoms and investigate the influence of the time $\tau_{r}$ during which the barrier is raised by increasing $I_{2}$ from zero to $3.9 \mathrm{~mA}$, well above the chemical potential. The final number squeezing $\xi^{2}$ is shown in Fig. 2. We observe a decrease of fluctuations below the shot noise limit with increasing $\tau_{r}$ up to $50 \mathrm{~ms}$, followed by a slow increase for longer times. This agrees with the expectation that an optimum should exist between very short $\tau_{r}$ creating excitations in the BEC and/or not leaving sufficient time for tunneling, and very long $\tau_{r}$ where heating and atom loss become important. For a quantitative analysis, we start by noting that low-energy excitations from the symmetric many-particle ground state correspond to populating the first excited mode, which is the longitudinal dipole mode for $V_{b}=0$ and becomes the Josephson plasmon mode as the barrier raises. These excitations are spaced by $\hbar \omega_{p}$, where the plasma frequency $\omega_{p}$ decreases from $\omega_{p}=\omega_{x}$ to 0 as the barrier raises [6]. The interesting dynamics can

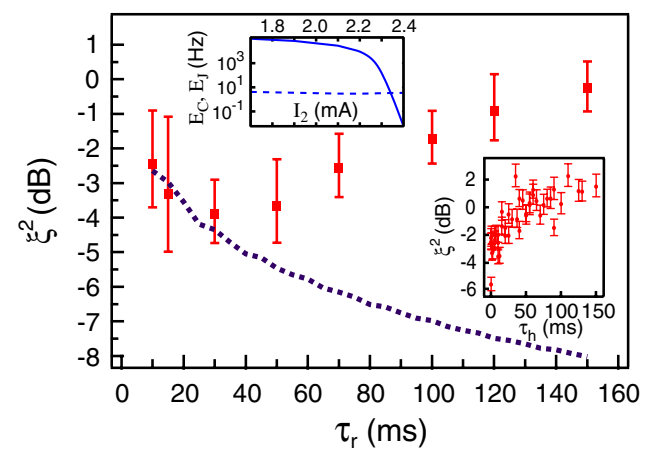

FIG. 2 (color online). First experiment. Fluctuations of the atom number difference as a function of splitting time $\tau_{r}$ for a BEC with $N=1300$. Red squares, measured $\xi^{2}$; dotted line, dynamical TMM simulation. Upper inset: $E_{C}$ (dashed line) and $E_{J}$ (solid line) from numerical 3D Gross-Pitaevskii equation solution for 1300 atoms using the full calculated potential. Lower inset: Degradation of the number squeezing under the influence of heating. The BEC is held for a variable time $\tau_{h}$ before splitting; $\tau_{r}=50 \mathrm{~ms}$ is constant. 


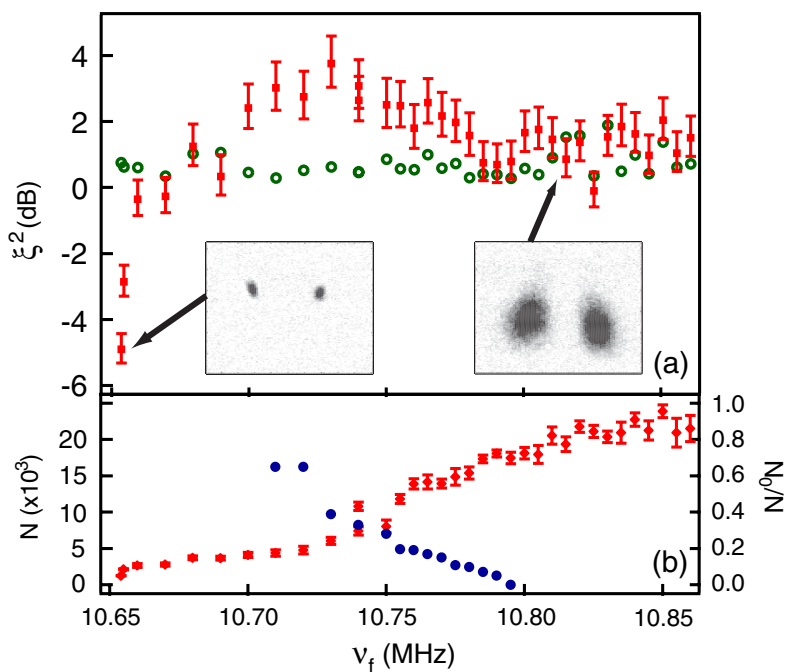

FIG. 3 (color online). Second experiment. (a) Effect of the temperature on $\xi^{2}$. We vary the final frequency $\nu_{f}$ of the evaporative cooling ramp before splitting the cloud using a $50 \mathrm{~ms}$ linear current ramp. Typical absorption images are shown in the inset of (a). Red squares, $\xi^{2}$; open green circles, fringe noise. (Estimation of the $\xi^{2}$ we expect to measure when the actual fluctuations are binomial-the estimate is obtained by analyzing a region of the image that does not contain atoms.) (b) Total atom number $N$ (red diamonds) and condensed fraction $N_{0} / N$ prior to splitting (blue circles).

be expected to occur when the barrier height approaches the chemical potential. In this region, $\hbar \omega_{p}$ is already much smaller than $\hbar \omega_{x}$, which is the energy scale of the next higher modes. Therefore, a TMM is expected to work well in this region at least as long as $k_{B} T<\hbar \omega_{x}$. The two parameters entering the TMM are the charging energy $E_{C}$ accounting for the interaction between the particles and the Josephson energy $E_{J}$ that characterizes the tunneling. We calculate them for each barrier height by solving the 3D Gross-Pitaevskii equation for the experimental trap [11]. In the TMM simulations, we describe the initial state before splitting by a thermal density matrix, which we evolve according to the von Neumann equation. We start the simulation at $I_{2}=1.9 \mathrm{~mA}$, slightly below the splitting point. We have checked that the results depend only weakly on the starting point (initial $I_{2}$ ) in this region. An initial temperature $T_{i}=50 \mathrm{nK}$ reproduces the measured fluctuations after a ramp of $10 \mathrm{~ms}$. The $T_{i}$ thus found is then used for all longer $\tau_{r}$. The simulation reproduces well the observed squeezing for $\tau_{r}$ up to $30 \mathrm{~ms}$. For $\tau_{r}>40 \mathrm{~ms}$, the experimental data show a degradation of squeezing which we attribute to technical heating. To confirm this hypothesis, we hold the BEC for a variable time $\tau_{h}$ before splitting it with a $50 \mathrm{~ms}$ ramp (inset of Fig. 2).

In a second experiment, we investigate the effect of the temperature on the squeezing. We ramp $I_{2}$ linearly from 0 to $3.9 \mathrm{~mA}$ in $50 \mathrm{~ms}$, and vary the temperature by changing the final frequency $\nu_{f}$ of the evaporative cooling ramp. The results are plotted in Fig. 3. Below $10.73 \mathrm{MHz}$, i.e., well below $T_{c}$, we observe a crossover from sub-binomial to superbinomial fluctuations with increasing temperature. Around $10.73 \mathrm{MHz}$, the condensed and thermal fractions are comparable, and we observe large super-Poissonian fluctuations with a maximum of $+3.8 \mathrm{~dB}$. As the temperature increases further, fluctuations decrease, and level when the condensate fraction reaches zero. The measured asymptotic level of $1 \mathrm{~dB}$ is consistent with binomial statistics given our fringe noise [Fig. 3(a), open green circles). According to the TMM calculation, our best result of $\xi^{2}=$ $-4.9_{-0.4}^{+0.5} \mathrm{~dB}$ corresponds to a phase coherence $\langle\cos \varphi\rangle \sim$ 0.93 immediately after the splitting, where $\varphi$ is the relative phase between the two clouds. This would result in a possible metrology gain of $-4.4 \mathrm{~dB}$ compared to the standard quantum limit using this state in an atom interferometer.

The observed sub-binomial and superbinomial regimes originate from the interplay between interactions and quantum statistics. Lowering the temperature, the onset of superbinomial fluctuations occurs when quantum degeneracy becomes important. Fluctuations are given by the probability distribution of the macroscopic configurations with a given atom number difference $n$. This distribution is binomial in the classical gas regime, leading to $\left\langle n^{2}\right\rangle=$ $N / 4$. In the degenerate regime, the entropy effect which favors small number differences vanishes and, if each Fock state with a given $n$ is equiprobable, $\left\langle n^{2}\right\rangle$ is as high as $N^{2} / 12$. The crossover from superbinomial to sub-binomial fluctuations comes from the interaction energy cost associated with number fluctuations, which eventually exceeds the available thermal energy. This low temperature regime is well described by the TMM, which predicts fluctuations $\left\langle n^{2}\right\rangle=k_{B} T / E_{C}$, as a result of the equipartition theorem. We heuristically extend the TMM to higher temperatures by assuming that the total fluctuations are the sum of the fluctuations of $N_{2 m}$ atoms in the condensate, which are described by the TMM, and $N-N_{2 m}$ atoms in a classical gas. This leads to $\left\langle n^{2}\right\rangle=k_{B} T / E_{c}+\left(N-N_{2 m}\right) / 4$. Here, $N_{2 m}$ has to be determined independently. This formula implies the existence of a maximum: on one hand the decrease of temperature increases $N_{2 m}$, which contributes to the superbinomial signal; on the other hand, interactions that tend to lower the fluctuations dominate more and more as the temperature decreases. Given that the maximum occurs at some fraction of the critical temperature $T_{c}$, where a macroscopic population in the two lowest mode appears, this maximum scales as $k_{B} T_{c} /\left(N E_{C}\right)$, which is large for a cold gas in the weakly interacting regime.

In order to account more accurately for the thermal cloud contribution, we perform a multimode calculation in the classical field approximation. The fluctuations are decomposed into a shot noise term $N$ plus a term where the fields appear in the normal order. We compute the latter by sampling the Glauber-P distribution that we approximate 


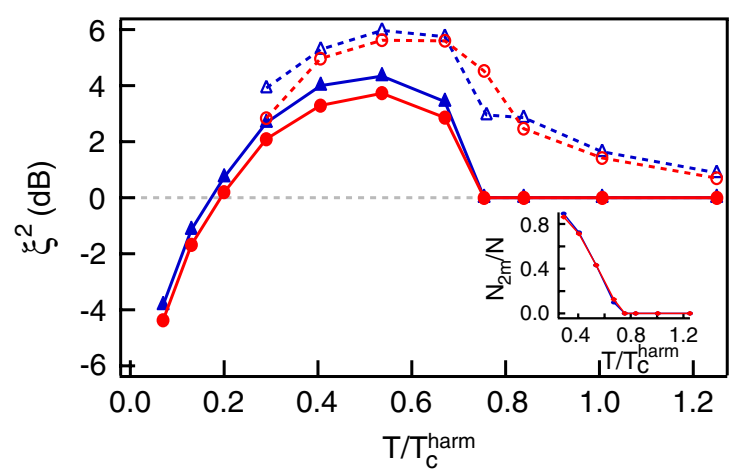

FIG. 4 (color online). Classical field simulations (open symbols and dotted lines) and modified TMM (full symbols and full lines). Dark gray (blue) lines and triangles, $N=6000$; light gray (red) lines and circles, $N=17000$. The trapping potential is harmonic transversally $\left(f_{y, z}=1120,1473 \mathrm{~Hz}\right)$, and quartic along $x$ with $V_{b} / h=2.69 \mathrm{kHz}$ and $x_{0}=3.8 \mu \mathrm{m}$. The longitudinal oscillation frequency within each well is $f_{x}=411 \mathrm{~Hz}$. The temperature unit in the figure is the ideal gas transition temperature in the harmonic potential prior to splitting $T_{c}^{\text {harm }}(N=6000$ : $\left.T_{c}^{\text {harm }}=0.89 \mu \mathrm{K} ; N=17000: T_{c}^{\text {harm }}=1.25 \mu \mathrm{K}\right)$. Inset: Fraction of the population in the two lowest energy modes $N_{2 m}$, extracted from the classical field simulations.

by the classical distribution $P \propto \exp \left\{-\beta E\left[\psi, \psi^{*}\right]\right\}$, where $E\left[\psi, \psi^{*}\right]$ is the Gross-Pitaevskii energy functional [15]. We include the first quantum correction to the classical field, which has the effect to change the shot noise term $N$ into $N-N_{2 m}$. As expected, the classical field approximation predicts an increase of fluctuations starting above $T_{c}$, when the gas enters the degenerate regime. The subbinomial regime is out of reach of the classical field approximation using the Glauber-P distribution. The results of both models are shown in Fig. 4. They exhibit a superbinomial maximum below $T_{c}$ like the experimental results, even though the latter were obtained in a dynamical process and with varying $N$. The inset shows $N_{2 m}$ extracted from the classical field calculations, which we also use in the TMM curve. In both models, fluctuations are largely independent of the atom number $N$ for a given condensed fraction, which explains why the experimental data have similar shape and maximum fluctuations in spite of the varying $N$.

These results show the interplay of quantum statistics and interactions in a simple and fundamental finitetemperature system. The double well can be seen as a "nutshell" version of the Bose-Hubbard model whose more complex dynamics also lead to the Mott insulator transition [16]. Our results also highlight the generic features of spatial splitters for trapped BECs: interactions allow for the creation of nonclassical states of potential interest for quantum metrology. However, the same interactions will lead to phase spreading with a rate propor- tional to $\xi \sqrt{N} E_{C}$ after the splitting. Hence, tuning the interactions to reduce $E_{C}$ after splitting is a necessary step for the use of such a nonlinear splitter in a real interferometer.

This work was funded in part by a EURYI grant. Our group is part of IFRAF. G. E. M. acknowledges support from the Hertz Foundation.

*Present address: Department of Physics, University of California, Berkeley, California 94720, USA.

${ }^{\dagger}$ Present address: Institut für Experimentalphysik, Heinrich Heine Universität, 40225 Düsseldorf, Germany.

jakob.reichel@ens.fr

[1] Y. Shin, M. Saba, T. A. Pasquini, W. Ketterle, D.E. Pritchard, and A.E. Leanhardt, Phys. Rev. Lett. 92, 050405 (2004).

[2] Y.-J. Wang, D.Z. Anderson, V. M. Bright, E. A. Cornell, Q. Diot, T. Kishimoto, M. Prentiss, R. A. Saravanan, S. R. Segal, and S. Wu, Phys. Rev. Lett. 94, 090405 (2005).

[3] T. Schumm, S. Hofferberth, L. M. Anderson, S. Wildermuth, S. Groth, I. Bar-Joseph, J. Schmiedmayer, and P. Krüger, Nature Phys. 1, 57 (2005).

[4] M. Albiez, R. Gati, J. Fölling, S. Hunsmann, M. Cristiani, and M. K. Oberthaler, Phys. Rev. Lett. 95, 010402 (2005).

[5] E. M. Wright, D. F. Walls, and J. C. Garrison, Phys. Rev. Lett. 77, 2158 (1996); J. Javanainen and M. Wilkens, Phys. Rev. Lett. 78, 4675 (1997); M. Lewenstein and L. You, Phys. Rev. Lett. 77, 3489 (1996); Y. Castin and J. Dalibard, Phys. Rev. A 55, 4330 (1997).

[6] A. J. Leggett and F. Sols, Phys. Rev. Lett. 81, 1344 (1998).

[7] M. Kitagawa and M. Ueda, Phys. Rev. A 47, 5138 (1993).

[8] G.-B. Jo, Y. Shin, S. Will, T. A. Pasquini, M. Saba, W. Ketterle, D. E. Pritchard, M. Vengalattore, and M. Prentiss, Phys. Rev. Lett. 98, 030407 (2007).

[9] J. Sebby-Strabley, B. L. Brown, M. Anderlini, P. J. Lee, W. D. Phillips, J. V. Porto, and P. R. Johnson, Phys. Rev. Lett. 98, 200405 (2007).

[10] J. Estève, C. Gross, A. Weller, S. Giovanazzi, and M. Oberthaler, Nature (London) 455, 1216 (2008).

[11] D. Ananikian and T. Bergeman, Phys. Rev. A 73, 013604 (2006), and references therein.

[12] J. Estève, J.-B. Trebbia, T. Schumm, A. Aspect, C. I. Westbrook, and I. Bouchoule, Phys. Rev. Lett. 96, 130403 (2006).

[13] G. Reinaudi, T. Lahaye, Z. Wang, and D. Guéry-Odelin, Opt. Lett. 32, 3143 (2007).

[14] $z_{p}=\sqrt{\left[f_{R}^{2}\left(\delta N_{L}\right)^{2}+f_{L}^{2}\left(\delta N_{R}\right)^{2}\right] /\left[f_{L} f_{R}\left(N_{L}+N_{R}\right)\right]}$, where $\delta N_{L}, \delta N_{R}$ are the atom number uncertainties in each well due to photon shot noise.

[15] A. Sinatra, Y. Castin, and Yun Li, Phys. Rev. A 81, 053623 (2010).

[16] M. Greiner, O. Mandel, T. W. Hänsch, and I. Bloch, Nature (London) 415, 39 (2002). 